\title{
Ab Initio Studies of the Reaction of Hydrogen Transfer from DNA to the Calicheamicinone Diradical
}

\author{
Anne-Marie Sapse, ${ }^{1,2}$ Robert Rothchild, ${ }^{1}$ Rohtash Kumar, ${ }^{3}$ and J. William Lown ${ }^{3}$ \\ ${ }^{1}$ City University of New York, John Jay College and Graduate Center \\ ${ }^{2}$ Rockefeller University, New York, NY, USA \\ ${ }^{3}$ Department of Chemistry, University of Alberta, Edmonton, Alberta, CA \\ Accepted November 27, 2001
}

\begin{abstract}
Background: The biological activity of enediyne chemotherapeutic (anti-cancer) agents is attributed to their ability to cleave duplex DNA. Part of the reaction of cleavage is the abstraction of hydrogens from the deoxyribose moiety of DNA by the biradical formed via a Bergman rearrangement. Methods: The mechanism of the reaction of abstraction of two hydrogen atoms from two deoxyribophosphate molecules by the calicheamicinone biradical is studied with $a b$ initio calculations at Hartree-Fock and post-Hartree-Fock level. The Titan program is used to perform the calculations.
\end{abstract}

Results: It is found that the reactions are exothermic and thus thermodynamically reasonable.

Conclusions: The mechanism of DNA cleavage by the enediyne-containing drugs is likely to proceed by the abstraction of the hydrogens from deoxyribose by the biradical formed by the drug. Further studies should determine in which way the modification of the drug's structure would make this reaction even more exothermic and, thus, more likely to occur.

\section{Introduction}

Modern strategies for the treatment of cancer using cytotoxic chemicals are generally based on the concept of prodrug activation, where the injected drug is a conjugate, acted upon by enzymes in the body in order to release the active form of the drug. Provided that the prodrugs can be delivered selectively to the tumor cells, for example by monoclonal antibodies, toxic effects during transport within the body would be minimized. On the other hand, if the activation of the drug would only be possible in tumor cells, a delivery device would be unnecessary. Still another similar concept relies on so-called ADEPT strategy (antibody dependent enzyme-mediated prodrug therapy) (1). The later requires that the prodrug is activated by an enzyme which has been delivered selectively to tumor cells by antibodies. In order to implement such strategies, suitable prodrugs have to be available which can be furnished with the various trigger devices. In this regard, several recently developed potent antitumor antibodies containing (Z)enediynes or related systems, represent promising

Address correspondence and reprint requests to: Anne-Marie Sapse, John Jay College, 445 W 59th St. NY, NY, 10019, USA. Phone: (212) 237-8949; fax: (212) 935-3594;

e-mail: acransg6@AOL.com. lead compounds. They are of interest, not only because of their unusual structures, but also because of their novel mode of action (2-6). Typically, they are composed of three functional units: a) an enediyne which is a part of a 10-membered ring, b) a trigger mechanism which, through a chemical reaction, leads to a reactive enediyne and c) a moiety which is responsible for the molecular recognition of DNA.

The biological activity of these compounds is attributed to their ability to cleave duplex DNA irreversibly, by the cyclization of the enediynes to afford aromatic compounds through the intermediacy of 1,4-dehydrobenzene biradicals, obtained via a Bergman rearrangement, as shown in Scheme 1. In general, DNA cleavage occurs in four phases:

1. Recognizing and binding to DNA by a specific structural feature covalently bonded or complexed to the enediyne.

2. Activation of the enediyne towards a Bergman rearrangement.

3. Bergman cyclization to give 1,4-dehydroarene biradicals.

4. Abstraction of hydrogen from DNA by the 1,4dehydroarene biradicals thereby inflicting permanent damage to the genetic material. 


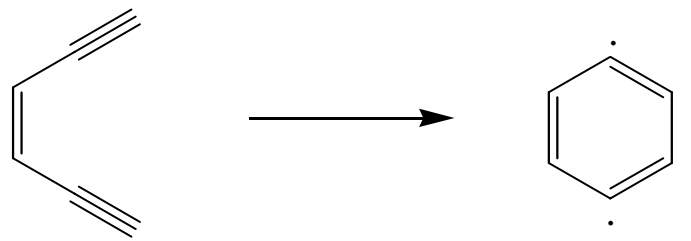

Scheme 1.

A number of theoretical studies have tried to determine the structure of some of these compounds as well as their mode of action. They comprise PRDDO studies of calicheamicin and esperamicin enediyne moieties (7), density functional studies (8) and solvated molecular dynamics simulations (9). Our group applied $a b$ initio and semi-empirical methods to the study of calicheamicinone and its undergoing the Bergman rearrangement $(10,11)$. Esperamicin Al, calicheamicin $\gamma \mathrm{l}$ and other enediynes such as dynamicin A and Neocarzinostatin chromophore are shown in Rothchild et al. (10).
Recently, the Janda group (12) proposed an alternative mechanism for the enediyne cyclization. They designed and synthesized a catalytic antibody that accelerates the Bergman cyclization of an enediynol substrate. Enediynol reacts to form a quinone by an oxidative route in aqueous buffer either in the presence, or absence, of a catalytic antibody. The proposed mechanism suggests that, at least in the presence of oxygen, it may be quinone formation rather than diradical formation that accounts for the DNA-cleaving ability of enediynes. However, Hamann and Wang (13) have carefully evaluated the "quinone" mechanism proposed by Janda et al. (12) and strongly support the conventional mechanism consisting of hydrogen transfer from the deoxyribose moiety of DNA to the calicheamicin diradical resulting from the Bergman cycloaromatization product. The mechanism of this reaction has been proposed by Ellestad et al. (14), and it is shown in Scheme 2. The diradical 1 becomes the monoradical 4 or 5, according to Path 1 or Path 2, by abstracting a hydrogen from compound 2 . The deoxyribose monoradicals thus obtained (compounds 3

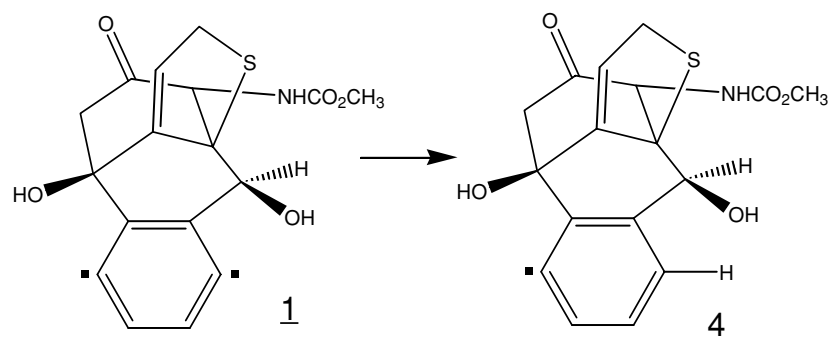

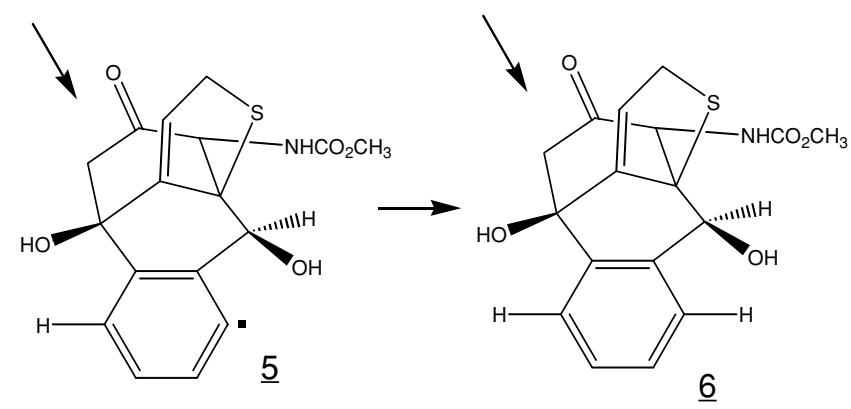

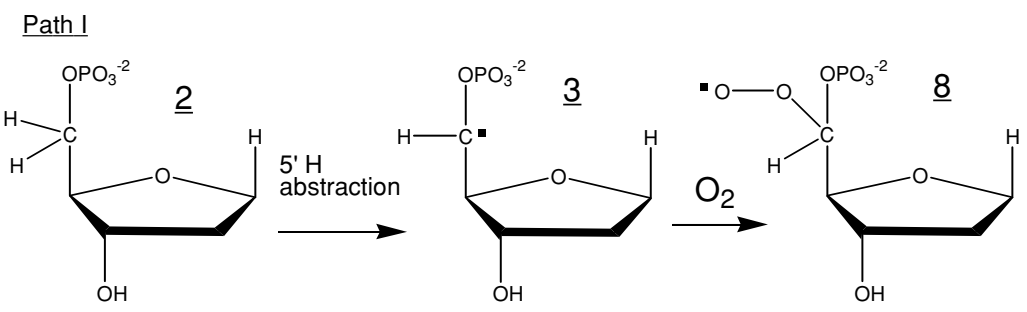

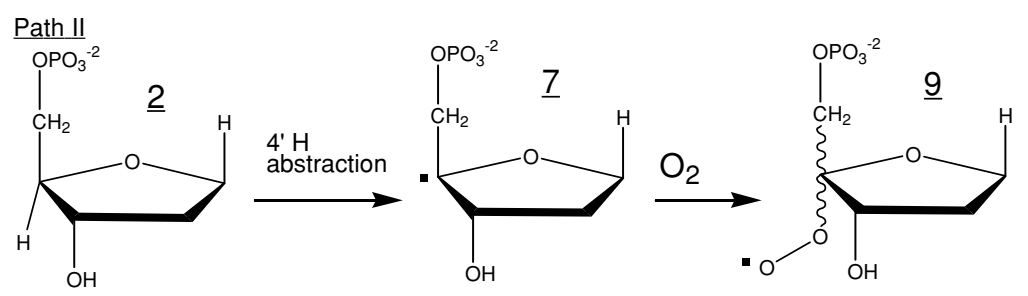


Table 1. The optimized energies of the molecules shown in Scheme 2. (au) Molecule

\begin{tabular}{|c|c|c|}
\hline \multirow[b]{3}{*}{ Calicheamicinone diradical (1) } & \multicolumn{2}{|c|}{ Energy } \\
\hline & $\mathrm{HF} / 6-31 \mathrm{G}^{*}$ & LMP2/6-31G*//HF/6-31G* \\
\hline & $\begin{array}{c}M=1 \\
-1478.51701\end{array}$ & $\begin{array}{c}M=3 \\
-1481.81614\end{array}$ \\
\hline Deoxyribose phosphate (2) & $\begin{array}{c}M=1 \\
-1060.58816\end{array}$ & $\begin{array}{c}M=1 \\
-1062.53345\end{array}$ \\
\hline Deoxyribose phosphate monoradical (3) & $\begin{array}{c}M=2 \\
-1059.95348\end{array}$ & $\begin{array}{c}M=2 \\
-1061.89308\end{array}$ \\
\hline Calichaemicinone monoradical (4) & -1479.28075 & -1482.48727 \\
\hline Calicheamicinone monoradical (5) & -1479.28211 & -1482.48919 \\
\hline Calicheamicinone $(6)$ & $\begin{array}{c}M=1 \\
-1479.93989\end{array}$ & $\begin{array}{c}M=1 \\
-1483.15674\end{array}$ \\
\hline Deoxyribose phosphate monoradical (7) & $\begin{array}{c}M=2 \\
-1059.95809\end{array}$ & $\begin{array}{c}M=2 \\
-1061.90023\end{array}$ \\
\hline \multicolumn{3}{|l|}{ Peroxydeoxyribose phosphate } \\
\hline $\begin{array}{r}\text { Monoradical (8) }(\mathrm{R}) \\
(\mathrm{S})\end{array}$ & $\begin{array}{l}-1209.59728 \\
-1209.59603\end{array}$ & $\begin{array}{l}-1211.88951 \\
-1211.88841\end{array}$ \\
\hline \multicolumn{3}{|l|}{ Peroxydeoxyribose phosphate } \\
\hline $\begin{array}{r}\text { Monoradical (9) }(\mathrm{R}) \\
\text { (S) }\end{array}$ & $\begin{array}{l}-1209.59800 \\
-1209.60854\end{array}$ & $\begin{array}{l}-1211.89089 \\
-1211.90442\end{array}$ \\
\hline $\mathrm{O}_{2}$ & $\begin{array}{c}M=3 \\
-149.59826\end{array}$ & $\begin{array}{c}M=3 \\
-149.94639\end{array}$ \\
\hline
\end{tabular}

and 7) react with an oxygen molecule, producing compounds 8 and 9 . The calicheamicinone monoradicals 4 or 5 gain another hydrogen atom from a second deoxyribose moiety and become compound 6.

Accordingly, the present study examines via $a b$ initio methods the energetics of these reactions, calculating the energies of each species.

\section{Methods and Results}

The Titan (15) computer program was used to perform quantum chemical ( $a b$ initio) calculations on all the molecules shown in Scheme 2. The two mechanisms shown in scheme 2 will be referred to as Path I and Path II, as indicated in the scheme.

The basis set used is $6-31 G^{*}$, which uses two Slater orbitals for the description of the valence electrons, one expanded in a series of three gaussian functions and one approximated by one gaussian, while the core electrons are described by one Slater orbital expanded in a series of six gaussians. In addition, this basis set sets $\mathrm{d}$ orbitals on the non-hydrogen atoms. The calculations are performed at Hartree-Fock level and at post-Hartree-Fock level. In order to include the correlation energy effects, the LMP2 method (local Moller-Plesset of second order) $(16,17)$ is applied, as implemented by the Titan program, also using the 6$31 G^{*}$ basis set. This energy is calculated as a single point, using the HF/6-31G* optimized geometry. The energy of the calicheamicinone biradical was calculated at both HF and LMP2 levels for the species featuring a spin multiplicity of three. In addition, the singlet species was also investigated. The monoradicals were all investigated with a spin multiplicity of two.

Table 1 lists the energies of all the entities from Scheme 2 at both HF/6-31G* and LMP2/6-31G*// HF/6-3 $1 \mathrm{G}^{*}$ levels. Table 2 lists the energies of the reactions present in Path I and Path II mechanisms.

Table 2. The energies (Kcals/mole) for reactions of Path I and Path II

\begin{tabular}{lcc}
\hline & \multicolumn{2}{c}{ Energy $^{\mathbf{a}}$} \\
\cline { 2 - 3 } Reaction & HF/6-31G* & $\begin{array}{c}\text { LMP2/6-31G*// } \\
\text { HF/6-31G* }\end{array}$ \\
\hline $1+2=3+4$ & 10.668 & 19.327 \\
$1+2=3+5$ & 11.546 & 20.519 \\
$1+2=7+4$ & 13.540 & 23.845 \\
$1+2=7+5$ & 14.495 & 25.037 \\
$3+\mathrm{O}_{2}=8$ & 28.142 & 27.177 \\
$7+\mathrm{O}_{2}=9$ & 32.467 & 36.230 \\
$1+2+2=6+3+3$ & 25.979 & 37.650 \\
$1+2+2=6+3+7$ & 28.928 & 42.105 \\
$1+2+2=6+7+7$ & 31.877 & 46.561 \\
& & \\
\hline
\end{tabular}

${ }^{a}$ These energies represent the number of Kilocalories per mole produced by the reactions listed, which are thus exothermic. 
The transition state of the migration of the hydrogen from the deoxyribose phosphate 2 to the diradical l was investigated with the AMI semiempirical method.

The energies shown in Table 2 do not take into consideration thermal factors, zero-point energies or entropies. The free energy change is thus approximated only by the change in energy. Given the large values of the energy changes and the fact that no large changes in entropy are to be expected by the reactions, this approximation should be valid.

Figs. 1-9 represent the optimized conformations of compounds 1 to 9 , in their lowest energy forms.

\section{Discussion}

Examining Tables 1-2, it is clear that the reactions leading to the transfer of hydrogen atoms from the deoxyribose entities to the calicheamicinone biradical are exothermic.

The calicheamicinone biradical can be either a triplet or a singlet. Accordingly, the calculations were performed for both species. As seen from Table 1, at both HF/6-31G* and LMP2/6-31G*//HF/6-31G* levels, the triplet, as expected, features a much lower energy than the singlet.

The drugs featuring the enediyne ring are attached to the DNA fragments via the long entities which can be either sugars or, in the case of the compounds studied by our group, a methyltrisulfur moiety. Our previous work shows the docking of the molecule into a DNA fragment (11). The enediyne ring undergoes the Bergman rearrangement after a sulfur attack on the ring, losing the moiety shown in Fig. 10 which is the moiety inserted into the minor groove of the DNA fragment. It is thus likely that the insertion takes place first, followed by the Bergman rearrangement. The biradical formed is in close proximity to the deoxyribose moieties of the two DNA strands and a hydrogen from each of them can be transferred to the biradical. As seen from Table 2, if both deoxyribose moieties lose the hydrogen via Path I, the reaction gives away a total energy of $25.979 \mathrm{Kcals} / \mathrm{mole}$ at HF level and of 37.650 Kcals/mole at LMP2 level. These energies increase if one of the deoxyriboses follows Path II, and increases even more if both react via Path II.

When the deoxyribose monoradical reacts with an oxygen molecule, again the reaction is exothermic, producing $28 \mathrm{Kcals} / \mathrm{mole}$ and $32 \mathrm{Kcals} / \mathrm{mole}$ at Hartree-Fock calculational level (Path I and Path II, respectively) and $27 \mathrm{Kcals} / \mathrm{mole}$ and $36 \mathrm{Kcals} / \mathrm{mole}$ at LMP2 calculational level. At both calculational levels, Path II is more exothermic than Path I.

It might be concluded that from a purely thermodynamic point of view, Path II should take precedence over Path I.

When the transition state of the reaction of the transfer of the hydrogen from the deoxyribophosphate

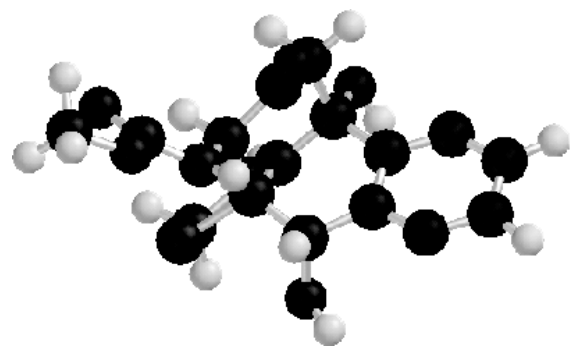

Fig. 1 .

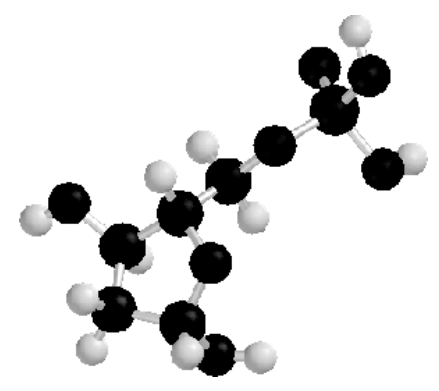

Fig. 2.

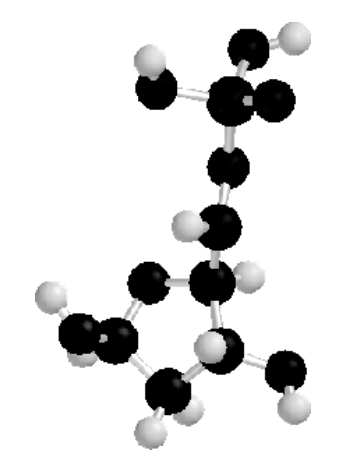

Fig. 3.

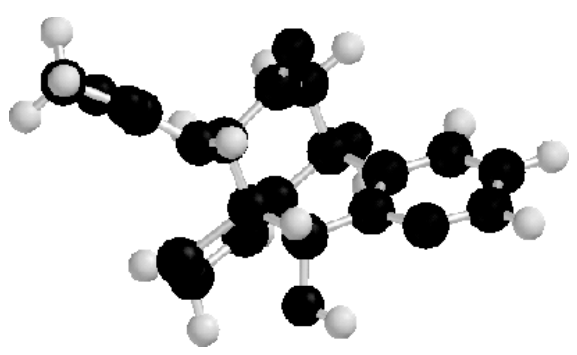

Fig. 4. 


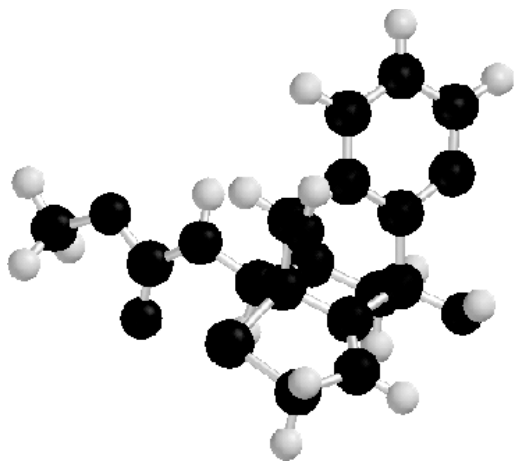

Fig. 5.

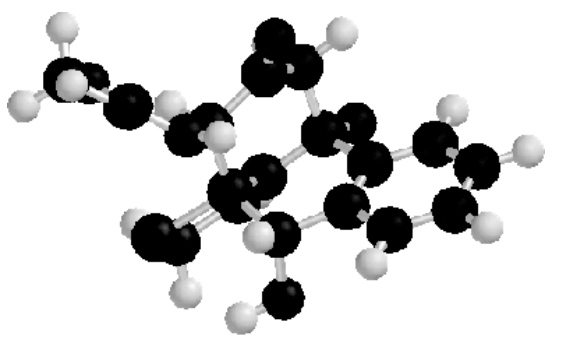

Fig. 6.

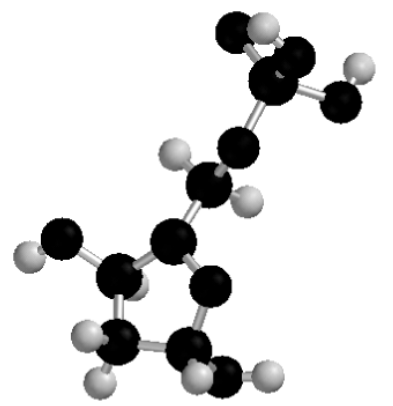

Fig. 7.

to the calicheamicinone biradical is investigated with AMI calculations (via Path I), it is found that the heat of formation of the transition state is $-457.941 \mathrm{Kcals} / \mathrm{mole}$. The distance from the hydrogen to the carbon on the deoxyribophosphate to which it was attached is $2.567 \mathrm{~A}$ and the $\mathrm{C}-\mathrm{H}$ bond length between the carbon on the calicheamicinone and the newly attached hydrogen is $1.105 \mathrm{~A}$. If the heat of formation is compared to the sum of the heats of formation of the calicheamicinone biradical and the deoxyribophosphate, which is of -484.200 Kcals/mole, it follows that the height of the transition state is of $26.253 \mathrm{Kcals} / \mathrm{mole}$. The same calculations performed for Path II shows a value of

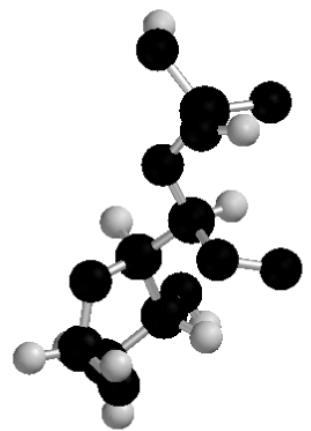

Fig. 8.

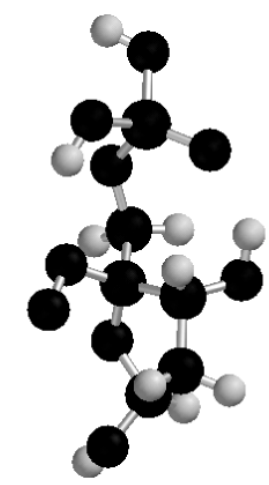

Fig. 9.

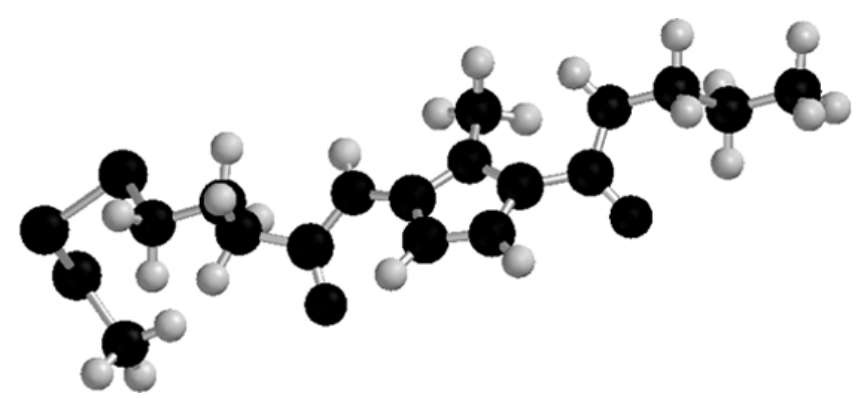

Fig. 10.

$-444.24 \mathrm{Kcals} / \mathrm{mole}$ for the transition state, with distances of $3.365 \mathrm{~A}$ and 1.104 A respectively, for carbon-to-hydrogen on the deoxyribophosphate and calicheamicinone. The height of this transition state is thus $39.96 \mathrm{Kcals} / \mathrm{mole}$, showing that Path II has to undergo a higher cost in energy to reach the transition state. However, the energy resulting from the reaction should be a more important parameter, especially since the calculations are performed at $a b$ initio level.

A point of interest is the fact that the ribophosphate monoradicals present a quasiplanar carbon where the unpaired electron is attached. Once the oxygen molecule is attached, that carbon becomes 
chiral and the attachment can result in obtaining either an $\mathrm{S}$ or an $\mathrm{R}$ isomer. These isomers are not equal in energy, due to the interactions with other groups in the molecules. These results, displayed in Table 1, show that for compound 8 the $\mathrm{R}$ isomer at the newly formed chiral center is more stable while for compound 9 the $S$ isomer is more stable. The reaction energies shown in Table 2 make use of the most stable species for 8 and 9.

\section{Acknowledgments}

The authors thank Dr. G. Ellestad for useful discussions. This investigation was partly supported by a grant (to J.W.L.) from the Natural Sciences and Engineering Research Council of Canada. Additional support was provided by the U.S. Education Department (to R.R.) and by the City University of New York PSC-CUNY Research Award Program (to AMS and R.R.)

\section{References}

1. Sedlacek HH, Hoffman D, Czech J, et al. (1991) The change in research for the therapy of tumors Chimia 45: 311-316.

2. Maier ME. (1995) Design of Enediyne prodrugs. Synlett 13-26.

3. Nicolaou KC. (1994) The magic of enediyne chemistry. Chem. Brit. 33-36.

4. Magnus P. (1994) General strategy using acetylene complexes for the synthesis of the enediyne antitumor agents esperamicin, calicheamicin , dynamicin and neocarzinostatin. Tetrahedron 50: 1397-1418.
5. Nicolaou KC, Dai WM. (1991) Chemistry and biology of the enediyne anticancer antibiotics. Angew. Chem. Int. 30: 1387-1416.

6. Goldber IH. (1991) Mechanism of neocarzinostatin action: role of DNA microstructure in determination of bistranded oxidative damage. Acc. Chem. Res. 24: 191-199.

7. Snyder JP. (1989) Cyclization of calicheamicin , esperamicin analogues: a predictive biradicaloid transition state. J. Am. Chem. Soc. 111: 7630-7632.

8. Lindh R, Ryde U, Schutz M. (1997) Density functional studies of enediynes. Theo. Chem. Acc. 97: 203.

9. Langley DR, GolicJ, KrishnanB, et al. (1994) The DNA esperamicin Al complex. A model based on solvated molecular dynamics simulations. J. Am. Chem. Soc. 116: 15-29.

10. Rothchild R, Sapse AM, Balkova A, Lown JW. (2000) Ab initio calculations on (-) calicheamicinone and some of its reactions involved in its activation and interaction with DNA. J. Biomolecular Structure and Dynamics 18: 413-421.

11. Sapse AM, Rothchild R, Lown JW. (2000) Semi-empirical, ab initio and molecular modeling studies on the DNA binding of a calicheamicinone-polyamide conjugate. J. Biomolecular Structure and Dynamics 18: 423-431.

12. Jones LH, Harwig CW, Wentworth P, et al. (2001) Conversion of enediynes into quinones by antibody catalysis and in aqueous buffers: implications for an alternative enediyne therapeutic mechanism. J. Am. Chem. Soc. 123: 3607-3608.

13. Hamann PR, Wang CP. (2001) Enediyne cyclization. Chem. Eng. News, Sept. 3, 8.

14. Ellestad G, Ding WD, Zein N, Townsend CA. (1995) DNA-Cleaving Properties of Calicheamicin $\gamma_{1}$. In: Enediyne Antibiotics as Antitumor Agents. Border DB, Doyle TW, eds., Marcel Dekker, New York, pp. 137-160.

15. Titan, Wavefunction, Inc, Irvine CA 92612.

16. Pulay P, Saebo S. (1986) Orbital invariant formulation and cond order gradient evaluation in Moller-Plesset perturbation theory. Theoretica Chimica Acta 69: 357-368.

17. Saebo S, Pulay P. (1993) Local treatment of electron correlation. Ann. Rev. Phys. Chem. 44: 213-236. 